\title{
El impacto social de un evento deportivo mediano recurrente: El caso del Maratón de Valencia
}

\author{
The social impact of a medium-size recurring sporting event: \\ The case of the Valencia Marathon
}

\section{David Parra Camacho, Sergio Aguado Berenguer, Mario Alguacil Jiménez}

Facultad de Ciencias de la Actividad Física y el Deporte. Universitat de València. España.

\author{
CORRESPONDENCIA:
}

David Parra Camacho

david.parra-camacho@uv.es

Recepción: septiembre 2020 • Aceptación: noviembre 2020
CÓMO CITAR EL ARTÍCULO:

Parra-Camacho, D., Aguado, S., \& Alguacil, M. (2021). El impacto social de un evento deportivo mediano recurrente: El caso del Maratón de Valencia. Cultura, Ciencia y Deporte, 16(50), 553-562. http://dx.doi.org/10.12800/ccd.v16i50.1576

\section{Resumen}

El objetivo de este estudio es analizar la percepción de los residentes de la ciudad de Valencia sobre el impacto social de la Maratón de Valencia Trinidad Alfonso e identificar los factores en los que se agrupan los indicadores de impactos percibidos. Se recogió una muestra de 391 residentes en la ciudad, mediante un muestreo de conveniencia, con una edad media de 34.26 (DT=11.78). El $56.5 \%$ eran hombres y un $43.5 \%$ eran mujeres. Se utilizaron 32 ítems, evaluados en una escala Likert de cinco puntos, sobre posibles beneficios y costes asociados a la celebración del evento en la ciudad. Se realizó un análisis factorial exploratorio que permitió identificar cinco factores: beneficios económicos e imagen de la ciudad (5 ítems), beneficios sociales (5 ítems), orgullo e identificación con la comunidad (3 ítems), impacto deportivo (4 ítems) y problemas socioeconómicos (5 ítems). Los residentes valoran positivamente el impacto del evento en aspectos relacionados con los beneficios económicos y la imagen de la ciudad, el orgullo y la identificación con la comunidad y el impacto en el deporte, mostrando una tendencia más neutral en los beneficios sociales y los problemas socioeconómicos. La práctica deportiva es una variable que influye significativamente en la percepción de los ciudadanos, mientras que el género o el nivel de estudios no parecen influir en la mayoría de los factores. Por último, a medida que aumenta la edad se observa una tendencia a valorar de forma más acusada los posibles problemas socioeconómicos derivados de la acogida del evento.

Palabras clave: Percepción de los residentes, impacto social, evento deportivo regular, evento deportivo mediano, percepción social

\section{Abstract}

The objective of this study is to analyze the perception of the residents of the city of Valencia on the social impact of the Trinidad Alfonso Valencia Marathon and to identify the factors in which the indicators of perceived impacts are grouped. A sample of 391 residents in the city was collected through a convenience sampling, with a mean age of 34.26 $(S D=11.78)$ and a distribution of $56.5 \%$ were men and $43.5 \%$ were women. Thirty-two items were used, evaluated on a five-point Likert scale, about possible benefits and costs associated with holding the event in the city. An exploratory factorial analysis was carried out that allowed identifying five factors: economic benefits and image of the city ( 5 items), social benefits (5 items), pride and identification with the community (3 items), sports impact (4 items), and socioeconomic problems ( 5 items). Residents positively value the event's impact on aspects related to economic benefits and city image, pride and identification with the community, and impact on sport, showing a more neutral trend in social benefits and socioeconomic problems. The practice of sport is a variable that significantly influences the perception of citizens, while gender or level of education does not seem to influence most factors. Finally, as age increases, there is a tendency to value more strongly the possible socioeconomic problems derived from hosting the event.

Key words: Residents' perception, social impact, regular sports event, medium-scale sports event, social perception 


\section{Introducción}

Los eventos deportivos son objeto de numerosas investigaciones desde diversas áreas de estudio desde su análisis económico, social y medioambiental, incluso desde otras áreas como la preparación física y nutricional (e.g., Martínez et al., 2019). Aunque la mayoría de los trabajos sobre el impacto social han centrado su interés en los mega eventos deportivos (campeonatos del mundo de fútbol y Juegos Olímpicos) (Chen et al., 2018), cada vez se observan más contribuciones sobre otros eventos deportivos de menor escala (e.g., Chen et al., 2018; Duan et al., 2020; Hautbois et al., 2020; Parra-Camacho et al., 2020; Sánchez-Sáez et al., 2018; Taks, 2013; Vegara-Ferri et al., 2020).

En este estudio se analiza el caso del Maratón Valencia Trinidad Alfonso del año 2015, que es un evento que se celebra anualmente y discurre por las calles y avenidas de la ciudad. En 2016 se le otorgó la medalla de oro (Gold Label) tras cumplir los requisitos de calidad marcados por la organización, situándolo así desde entonces entre los mejores del mundo (Maudos et al., 2020). En 2020 se ha convertido en el primer maratón de España en conseguir la Platinum Label, la cual solo tienen doce carreras en el mundo y en Europa únicamente los maratones de Berlín y Londres. La participación ha crecido en las últimas ediciones, con más de 25000 participantes en la edición de 2019, de los que un $20 \%$ procede del extranjero (Maudos et al., 2020).

Los mega eventos se refieren a los eventos más grandes y significativos que tienen la capacidad de generar un nivel sustancial de turismo, cobertura mediática, prestigio y beneficios financieros para la ciudad anfitriona (Chen et al., 2018). Por lo tanto, el resto de los eventos que no entran dentro de estas características podrían considerarse como eventos de pequeña o mediana escala. Antes de entrar en las características de los eventos de menor escala, debemos tener en cuenta que por impacto social de un evento deportivo podemos entender los cambios en la calidad de vida de los residentes como consecuencia de la acogida de un evento deportivo (Parra-Camacho et al., 2020).

De acuerdo con Higham (1999, p. 87) los eventos de menor escala incluirían a "las competiciones deportivas regulares (ligas de hockey sobre hielo, baloncesto, fútbol o rugby), instalaciones deportivas internacionales, competiciones nacionales, masters o deportes para discapacitados, y similares". De este modo, algunos eventos de menor escala atraen espectadores, mientras que otros, debido a sus características, como es el caso de las maratones, las carreras populares emblemáticas o triatlones, atraen participantes (Higham, 1999). Sin embargo, Gibson et al. (2003) señalan que es necesario conceptualizar los eventos de pequeña escala en términos relativos, ya que las definiciones indicadas antes se aplican igualmente a las competiciones deportivas con apoyo popular local y, al mismo tiempo, a los acontecimientos deportivos que atraen a espectadores y participantes a nivel nacional, e incluso, a nivel internacional. La distinción entre los eventos de menor tamaño y los mega-eventos no solo hace referencia al tamaño del acontecimiento, sino también a la necesidad de aportar menos recursos públicos.

$\mathrm{Al}$ igual que los mega eventos, es probable que los eventos recurrentes de menor escala estimulen efectos positivos y negativos en diversas dimensiones de la comunidad de acogida, incluidos los aspectos económicos, socioculturales y ambientales (Ouyang et al., 2019). Este tipo de acontecimientos puede requerir alguna mejora de las instalaciones deportivas existentes $y$, a veces, la construcción de una nueva instalación deportiva, pero por lo general no requieren grandes proyectos de infraestructura urbana como en los mega eventos (Taks, 2013). Por esta razón, se ha comprobado que los efectos económicos positivos generados por un acontecimiento de menor escala superan a los negativos (Ouyang et al., 2019), permitiendo generar, dada su periodicidad en el municipio, una corriente constante de visitantes y gastos (O’Brien \& Chalip, 2007). Los resultados de estudios previos indican que, al aumentar los ingresos procedentes del alojamiento y la alimentación, la celebración de eventos deportivos de menor escala proporciona beneficios económicos en los municipios que los acogen (Gibson et al., 2012; Veltri et al., 2009).

Los eventos deportivos regulares pueden contribuir a generar impactos socioculturales positivos relacionados con el sentimiento de pertenencia, identificación y orgullo de la comunidad (Ouyang et al., 2019), contribuyendo a mejorar el apego de la comunidad y, por lo tanto, pueden recibir recursos y apoyo público (Getz et al., 2012). Asimismo, el hecho de utilizar las instalaciones e infraestructuras existentes no solo contribuye a un menor gasto económico, sino también a la reducción del impacto en el medio ambiente (Daniels \& Norman, 2003; Ouyang et al., 2019; Veltri et al., 2009; Wilson, 2006).

Desde el punto de vista de las posibilidades de participación e involucración de la comunidad en el evento, los deportistas que participan en los mega eventos suelen ser de nivel de élite y los miembros de la comunidad de la ciudad anfitriona rara vez tienen la oportunidad de participar en ellos (Chen et al., 2018). También se puede esperar que las oportunidades de crecimiento personal y de desarrollo de las aptitudes de los residentes locales sean más altas en el contexto de eventos más pequeños, ya que existen más posibili- 
dades de participar en la planificación y organización que en los mega eventos deportivos (Taks, 2013). En este sentido, Taks (2013) concluye que existen razones para creer que los eventos de pequeña escala pueden ser más relevantes en la generación de beneficios duraderos para las comunidades anfitrionas en comparación con los mega eventos.

Por otro lado, la actividad física regular se ha convertido en una cuestión de política pública dados sus efectos en la salud. En este sentido, los eventos regulares participativos pueden estar en mejores condiciones de actuar como desencadenantes de la participación deportiva (ya que terminar un maratón puede representar una forma de logro que recompensa un entrenamiento físico exigente), así como ser un factor de mantenimiento de la actividad física después del evento (Hautbois et al., 2020).

Existen diversas teorías que tratan de explicar los resultados del impacto social de los eventos deportivos. En este estudio utilizamos la Teoría de Intercambio Social (TIS) como marco de referencia a la hora de analizar las percepciones de los residentes sobre el Maratón Valencia. Dicha teoría proviene de la investigación en el área del turismo, aplicándose a los eventos deportivos entendidos como un fenómeno turístico. La TIS señala que la percepción de los residentes locales sobre el desarrollo turístico es positiva cuando estas personas reciben más beneficios de la industria turística, y algunos residentes perciben el desarrollo turístico como negativo cuando perciben que incurren en más costes (Gursoy et al., 2019). Por lo tanto, aplicado al caso de los eventos deportivos podemos decir que si los residentes consideran que el evento genera más beneficios que costes para la comunidad tenderán a respaldar la celebración del evento (Gursoy et al., 2017; Waitt, 2003). Esta evaluación plantea un dilema social, ya que los residentes deben considerar si el evento puede ser positivo o negativo para la comunidad en la que residen en términos de intereses personales o colectivos (Chien et al., 2012).

Las aportaciones sobre los eventos de menor escala son escasas al compararlas con los trabajos realizados sobre los mega eventos. Algunos trabajos han analizado las relaciones entre los impactos percibidos y variables como el apoyo a la celebración del evento (Duan et al., 2020; Parra-Camacho et al., 2020), calidad de vida (Duan et al., 2020), satisfacción general de los residentes e intenciones futuras (Parra-Camacho et al., 2016; Vegara-Ferri et al., 2020). Estos trabajos han identificado algunos factores en los que se agrupan los indicadores asociados a los impactos de los eventos de menor escala. Por ejemplo, el estudio de Duan et al. (2020) agrupaba los indicadores en cuatro categorías: económicos, sociales, medioambientales y psicológicos. El trabajo de Vegara-Ferri et al. (2020) identificó cuatro factores que miden el constructo del impacto social en eventos deportivos de pequeña escala: beneficios socioeconómicos, beneficios socioculturales, beneficios deportivos y costes sociales. También, el trabajo de Parra-Camacho et al. (2020), diferenció entre participación deportiva e imagen de la ciudad, desarrollo social y capital humano y desarrollo económico. En el caso del estudio de Chen et al. (2018) sobre la percepción de un evento similar al analizado en este trabajo (Maratón de Hong Kong) los indicadores propuestos se agruparon en cuatro factores: impactos económicos y reconocimiento, desarrollo cultural, diversidad cultural, problemas sociales y problemas de tráfico y congestión. Otras aportaciones han clasificado los impactos sociales positivos en tangibles e intangibles según su naturaleza (Parra-Camacho et al., 2016).

Así pues, a partir de los estudios previos en esta área, en este trabajo se presentan una serie de indicadores que pretenden medir el impacto social de un evento deportivo que se celebra anualmente con un carácter participativo. Por lo tanto, el objetivo de este estudio es analizar el impacto social de un evento deportivo de carácter regular y participativo e identificar los factores en los que se agrupan los indicadores de impactos percibidos.

\section{Método}

\section{Participantes}

En este estudio participaron 391 residentes de la ciudad de Valencia con una edad media de 34.26 (DT $=11.78)$ con edades comprendidas entre los 18 y los 69 años. Según el género, el 56.5\% $(n=221)$ eran hombres mientras que un $43.5 \%(\mathrm{n}=170)$ eran mujeres. En función del nivel de estudios, el 21.7\% ( $\mathrm{n}=85)$ tiene estudios primarios, el $37.1 \%(n=145)$ tiene estudios secundarios y el $41.2 \%(n=161)$ tiene estudios universitarios.

\section{Instrumento}

Para consultar la percepción sobre el impacto social del evento se utilizó un instrumento compuesto por 32 ítems adaptados de estudios previos (Djaballah et al., 2015; Ntloko \& Swart, 2008; Parra-Camacho et al., 2016; Taks, 2013) sobre posibles beneficios y costes asociados a la celebración del evento en la ciudad. Todos los indicadores fueron evaluados mediante una escala de tipo Likert de cinco puntos ( $1=$ totalmente en desacuerdo; $5=$ totalmente de acuerdo). 


\section{Procedimiento}

Se utilizó un procedimiento de muestreo intencional no probabilístico de conveniencia, de acuerdo con lo realizado en otros trabajos en esta área (Oshimi \& Harada, 2019; Prayag et al., 2013). Tal y como señalan Kim et al. (2006), una de las principales debilidades asociadas al muestreo de conveniencia es el sesgo de selección. De este modo, para evitar este sesgo en el trabajo de campo, se indicó a los entrevistadores que recogieran encuestas de distintos grupos de población con la finalidad de entrevistar a colectivos proporcionales de residentes en función del género y la edad.

Para la distribución y recogida de los cuestionarios se contó con un equipo de entrevistadores formados en un seminario sobre aspectos generales de la investigación social y en la gestión del deporte. También se les explicó el objetivo y propósito del estudio, así como las características de los ítems y preguntas que componen el cuestionario. Los residentes entrevistados completaban el cuestionario de forma autoadministrada con presencia del investigador.

El estudio fue autorizado por el comité de ética de investigación en humanos de la Universitat de València dentro del proyecto de investigación "análisis de las percepciones de los impactos de los eventos deportivos" con número de procedimiento H1422448340492.

\section{Análisis estadísticos}

Los análisis estadísticos se realizaron con el programa SPSS v. 24 y el programa FACTOR para análisis factoriales. En primer lugar, para comprobar las propiedades psicométricas de los ítems se utilizaron los estadísticos de la media, desviación típica, asimetría y curtosis. Posteriormente se realizó un análisis factorial exploratorio (AFE) a partir de las recomendaciones de Lloret-Segura et al. (2014). Este análisis se realizó mediante el método de extracción de Máxima Verosimilitud (MV) y se utilizó el método de rotación oblimin directa. Para determinar el número de factores se utilizó el procedimiento de optimización implementada del análisis paralelo (Timmerman \& Lorenzo-Seva, 2011), mientras que para comprobar el ajuste del modelo se analizaron los coeficientes de la Raíz Media Cuadrática Residual (RMCR) y el índice gamma o de bondad de ajuste (GFI) propuesto por Tanaka y Huba (1989). Por otro lado, los ítems con cargas factoriales inferiores a .40 o superiores a este valor en dos o más factores se eliminaron antes de realizar el siguiente AFE. Por último, se comprobó la interpretabilidad teórica de la solución factorial extraída del AFE, así como la fiabilidad de los factores extraídos mediante el alfa de Cronbach (Hair et al., 2014).
Para comprobar la influencia de las variables sociodemográficas se utilizaron las pruebas t para muestras independientes y la prueba ANOVA de un factor, con aplicación previa de la prueba de homogeneidad de las varianzas. Para comprobar el tamaño del efecto se utilizó la d de Cohen y el valor de eta al cuadrado. Las diferencias entre categorías se analizaron mediante la prueba de Bonferroni. Por último, se utilizó la correlación de Pearson para comprobar la asociación entre la edad y las puntuaciones medias de los factores de impactos percibidos.

\section{Resultados}

\section{Análisis descriptivo}

En la tabla 1 se muestran la media, desviación típica, asimetría y curtosis de cada indicador. La mayoría de los indicadores presentan valores superiores al valor tres de la escala Likert, que indicaría una tendencia de los residentes hacia al acuerdo, destacando los relacionados con los beneficios económicos, la oportunidad para el entretenimiento, el incremento del orgullo de los residentes por acoger el evento, la mejora de la reputación del municipio como destino de eventos deportivos o la capacidad del municipio para acoger eventos deportivos.

Los indicadores con puntuaciones más reducida son los relacionados con "los beneficios de la celebración del evento se distribuyen de forma equilibrada en la sociedad valenciana", "permite promocionar e incrementar el conocimiento sobre los clubes deportivos locales" y "fomenta la inclusión de grupos desfavorecidos o en riesgo de exclusión social". Por otro lado, los valores de asimetría y curtosis son aceptables ya que son inferiores a 3.0 en todos los ítems (Chou \& Bentler, 1995).

\section{Análisis factorial exploratorio}

Para comprobar la agrupación de los indicadores por factores se realizó un AFE con los 32 ítems de la escala. El análisis paralelo recomendó la agrupación de los indicadores en cinco factores, permitiendo una interpretación teórica adecuada de los factores identificados. Siguiendo las recomendaciones de Lloret et al. (2014), se eliminaron diez ítems que presentaban cargas factoriales reducidas o cruzadas por encima del punto de corte establecido (.40). Los factores identificados y los indicadores que los integran se muestran en la tabla 2. Los indicadores eliminados fueron los siguientes: 5, 7, $8,10,13,17,18,20,31$ у 32 . 
Tabla 1. Media, desviación típica, asimetría y curtosis de los indicadores de los impactos percibidos por los residentes sobre el Maratón de Valencia.

\begin{tabular}{|c|c|c|c|c|}
\hline Item & Media & DT & Asimetría & Curtosis \\
\hline 1. Genera beneficios económicos para el municipio & 3.72 & .83 & .12 & -.86 \\
\hline 2. Ayuda a generar oportunidades para trabajar & 3.28 & .82 & -.14 & -.29 \\
\hline 3. Atrae turistas al municipio & 3.57 & .77 & -.10 & -.16 \\
\hline 4. Es una oportunidad para los comercios y los negocios locales & 3.66 & .75 & -.19 & -.04 \\
\hline 5. La celebración del evento contribuye a incrementar el gasto público para el deporte & 3.38 & .89 & -.15 & -.26 \\
\hline 6. Los beneficios de la celebración del evento se distribuyen de forma equilibrada en el municipio & 2.57 & .88 & .57 & .20 \\
\hline 7. Es una oportunidad para el entretenimiento de los residentes & 3.70 & .83 & -.17 & -.26 \\
\hline 8. Ofrece una oportunidad para divertirse con la familia y los amigos & 3.60 & .72 & -.24 & -.14 \\
\hline 9. Proporciona una oportunidad para conocer a nuevas personas & 3.33 & .83 & -.45 & -.21 \\
\hline 10. Contribuye a mejorar la hospitalidad y la solidaridad de los residentes con los visitantes & 3.40 & .75 & .03 & -.15 \\
\hline 11. El evento contribuye a la inclusión de personas con discapacidad & 3.11 & .80 & .04 & -.16 \\
\hline 12. Fomenta la inclusión de grupos desfavorecidos o en riesgo de exclusión social & 3.09 & .81 & -.28 & -.53 \\
\hline 13. Incrementa el orgullo de los residentes por ser los anfitriones del evento & 3.71 & .85 & -.36 & .06 \\
\hline 14. Contribuye a que los residentes se sientan bien consigo mismos y con el conjunto de la sociedad & 3.47 & .79 & -.28 & -.16 \\
\hline 15. Incrementa el sentimiento de pertenencia al municipio en el que reside & 3.44 & .84 & .00 & -.34 \\
\hline 16. Incrementa la sensación de bienestar y mejora la calidad de vida de los residentes & 3.35 & .86 & -.16 & -.24 \\
\hline $\begin{array}{l}\text { 17. Los residentes tienen la oportunidad de participar en la planificación y organización del evento } \\
\text { (como voluntarios, trabajadores...) }\end{array}$ & 3.10 & .84 & -.17 & -.49 \\
\hline $\begin{array}{l}\text { 18. Incrementa las habilidades y el conocimiento de los residentes sobre la organización de eventos } \\
\text { deportivos }\end{array}$ & 3.21 & .98 & -.03 & -.56 \\
\hline 19. Muestra la capacidad del municipio para acoger eventos deportivos & 4.05 & .77 & -.56 & .20 \\
\hline $\begin{array}{l}\text { 20. Contribuye al desarrollo de redes de voluntariado que pueden ser útiles para otros } \\
\text { acontecimientos que se celebren en el municipio }\end{array}$ & 3.58 & .75 & -.24 & .34 \\
\hline 21. Mejora la reputación del municipio como destino de eventos deportivos & 4.05 & .81 & -.52 & -.29 \\
\hline 22. Promociona el deporte entre los más jóvenes & 3.57 & .80 & -.29 & -.07 \\
\hline 23. Incrementa el interés por la práctica de deporte y actividad física entre los residentes & 3.49 & .89 & -.03 & -.45 \\
\hline 24. Fomenta la participación de la mujer en el deporte & 3.37 & .91 & .03 & -.31 \\
\hline 25. Permite promocionar e incrementar el conocimiento sobre los clubes deportivos locales & 2.91 & .85 & .03 & -.14 \\
\hline 26. Causa alteraciones en la vida diaria de los residentes (ruido, tráfico, congestión...) & 3.41 & .77 & -.17 & .03 \\
\hline 27. Restringe el acceso de los residentes a espacios o instalaciones públicas & 3.08 & .87 & -.06 & -.43 \\
\hline 28. Está asociado con la aglomeración o acumulación desordenada de personas & 3.36 & .87 & -.26 & .14 \\
\hline 29. Incrementa el gasto público asociado a los eventos & 2.98 & .77 & -.10 & .25 \\
\hline $\begin{array}{l}\text { 30. Provoca un incremento de los precios de algunos productos como la comida y/o servicios en el } \\
\text { municipio }\end{array}$ & 2.73 & .96 & -.03 & -.74 \\
\hline 31. Provoca daños en el medioambiente (contaminación, basura...) & 2.80 & 1.11 & .05 & -.74 \\
\hline 32. Incrementa el riesgo de la ciudad de ser objetivo del terrorismo & 2.72 & 1.12 & .04 & -.87 \\
\hline
\end{tabular}

Nota: DT= Desviación típica.

Para comprobar el ajuste del modelo se analizaron los coeficientes de la Raíz Media Cuadrática Residual (RMCR) y el índice gamma o GFI, que mostraron valores dentro de los puntos de corte recomendados: $\mathrm{RMCR}=.04$ (<.50) GFI=.98 (>.95). La varianza explicada por los 22 ítems agrupados en los tres factores fue del 54.32\%. El coeficiente del alfa de Cronbach osciló entre .61 y .79 según el factor.

\section{Análisis descriptivo de los factores}

En la tabla 3 se muestran las medias y desviaciones típicas de cada factor, observándose una tendencia positiva en el factor de beneficios económicos e imagen de la ciudad, el factor de orgullo e identificación con la comunidad y la dimensión de impacto deportivo. El factor de beneficios sociales y el de problemas socioeconómicos, presentan valores cercanos al valor neutral de la escala.

\section{Impacto de las variables sociodemográficas}

A continuación, se muestran los resultados de la influencia de diversas variables sociodemográficas en la percepción sobre los impactos. En primer lugar, se observó que en función del género (ver tabla 4) existían diferencias estadísticamente significativas $(\mathrm{p}<.05)$ solo para la percepción sobre los beneficios económicos e 
Tabla 2. Estructura factorial rotada de la escala sobre la percepción de los residentes en torno a los impactos de la Maratón de Valencia, comunalidades, autovalores y varianza explicada.

\begin{tabular}{|c|c|c|c|c|c|c|}
\hline & F1 & F2 & F3 & F4 & F5 & Com. \\
\hline \multicolumn{7}{|l|}{ Factor 1 - Beneficios económicos e imagen de la ciudad } \\
\hline 1. Genera beneficios económicos para el municipio & .45 & & & & & .23 \\
\hline 3. Atrae turistas al municipio. & .68 & & & & & .48 \\
\hline 4. Es una oportunidad para los comercios y los negocios locales & .54 & & & & & .32 \\
\hline 19. Muestra la capacidad del municipio para acoger eventos deportivos & .47 & & & & & .40 \\
\hline 21. Mejora la reputación del municipio como destino de eventos deportivos & .52 & & & & & .48 \\
\hline
\end{tabular}

\section{Factor 2 - Beneficios sociales}

2. Ayuda a generar oportunidades para trabajar

6. Los beneficios de la celebración del evento se distribuyen de forma equilibrada en el municipio

9. Proporciona una oportunidad para conocer a nuevas personas

11. El evento contribuye a la inclusión de personas con discapacidad

12. Fomenta la inclusión de grupos desfavorecidos o en riesgo de exclusión social

Factor 3 - Orgullo e identificación con la comunidad

14. Incrementa el orgullo de los residentes por ser los anfitriones del evento

15. Contribuye a que los residentes se sientan bien consigo mismos y con el conjunto de la sociedad

16. Incrementa el sentimiento de pertenencia al municipio en el que reside

\section{Factor 4 - Impacto deportivo}

22. Promociona el deporte entre los más jóvenes

23. Incrementa el interés por la práctica de deporte y actividad física entre los residentes

24. Fomenta la participación de la mujer en el deporte

25. Permite promocionar e incrementar el conocimiento sobre los clubes deportivos locales

\section{Factor 5 - Problemas socioeconómicos}

26. Causa alteraciones en la vida diaria de los residentes (ruido, tráfico, congestión...)

27. Restringe el acceso de los residentes a espacios o instalaciones públicas

28. Está asociado con la aglomeración o acumulación desordenada de personas

29. Incrementa el gasto público asociado a los eventos

30. Provoca un incremento de los precios de algunos productos como la comida y/o servicios en el municipio

\section{Eigenvalue}

Variance Explained (\%)

Items

Alfa de Crombach

$\begin{array}{lllll}7.73 \% & 6.20 \% & 11.26 \% & 20.26 \% & 8.86 \%\end{array}$

$\begin{array}{lllll}5 & 5 & 3 & 4 & 5\end{array}$

$\begin{array}{lllll}.69 & .61 & .74 & .79 & .66\end{array}$

Nota: Com.=Comunalidad

imagen de la ciudad, observándose en los hombres una media ligeramente superior a las mujeres.

En segundo lugar, se comprobó la influencia del nivel de estudios en la percepción social (ver tabla 5). Se observaron diferencias estadísticamente significativas $(p<.05)$ en el factor de impacto deportivo, entre el grupo con un nivel de estudios primarios y el grupo con un nivel de estudios universitarios.

Se comprobó el impacto de la práctica deportiva relacionada con el evento, es decir, el running o el atletismo, en la percepción sobre los impactos del Maratón. En la tabla 6 se puede observar que los que practican esta ac-
Tabla 3. Media y desviación típica de los factores sobre impactos percibidos por los residentes sobre la Maratón de Valencia.

\begin{tabular}{lcc}
\hline Item & Media & DT \\
\hline $\begin{array}{l}\text { Factor 1 - Beneficios económicos e imagen de } \\
\text { la ciudad }\end{array}$ & 3.81 & .52 \\
$\begin{array}{l}\text { Factor 2 - Beneficios sociales } \\
\text { Factor 3 - Orgullo e identificación con la } \\
\quad \begin{array}{l}\text { comunidad } \\
\text { Factor 4 - Impacto deportivo }\end{array}\end{array}$ & 3.08 & .52 \\
Factor 5 - Problemas socioeconómicos & 3.34 & .68 \\
\hline
\end{tabular}

Nota: DT= Desviación típica. 
Tabla 4. Influencia del género en la percepción sobre los impactos de la Maratón.

\begin{tabular}{|c|c|c|c|c|c|}
\hline & $\begin{array}{c}\text { Hombres } \\
\text { M (DT) }\end{array}$ & $\begin{array}{c}\text { Mujeres } \\
\text { M (DT) }\end{array}$ & $\mathbf{T}$ & P valor & d Cohen \\
\hline Factor 1 - Beneficios económicos e imagen de la ciudad & $3.89(.50)$ & $3.72(.54)$ & 3.22 & $.001 * * *$ & .33 \\
\hline Factor 2 - Beneficios sociales & $3.08(.49)$ & $3.08(.56)$ & -.07 & .94 & - \\
\hline Factor 3 - Orgullo e identificación con la comunidad & $3.44(.62)$ & $3.40(.74)$ & .56 & .57 & - \\
\hline Factor 4 - Impacto deportivo & $3.36(.67)$ & $3.31(.70)$ & .77 & .44 & - \\
\hline Factor 5 - Problemas socioeconómicos & $3.08(.50)$ & $3.14(.62)$ & -.99 & .32 & - \\
\hline
\end{tabular}

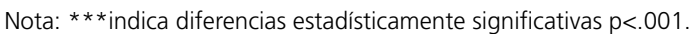

Tabla 5. Influencia del nivel de estudios en la percepción sobre los impactos de la Maratón.

\begin{tabular}{|c|c|c|c|c|c|c|}
\hline & $\begin{array}{c}\text { Primarios } \\
\text { M (DT) }\end{array}$ & $\begin{array}{l}\text { Secundarios } \\
\text { M (DT) }\end{array}$ & $\begin{array}{l}\text { Universitarios } \\
\text { M (DT) }\end{array}$ & $\mathbf{F}$ & P valor & d Cohen \\
\hline Factor 1 - Beneficios económicos e imagen de la ciudad & $3.76(.56)$ & $3.80(.53)$ & $3.84(.50)$ & .82 & .44 & - \\
\hline Factor 2 - Beneficios sociales & $3.06(.54)$ & $3.06(.52)$ & $3.12(.50)$ & .74 & .48 & - \\
\hline Factor 3 - Orgullo e identificación con la comunidad & $3.27(.63)$ & $3.44(.68)$ & $3.48(.69)$ & 2.65 & .07 & - \\
\hline Factor 4 - Impacto deportivo & $3.18(.69)$ & $3.34(.66)$ & $3.42(.68)$ & 3.50 & $.03 *$ & .02 \\
\hline Factor 5 - Problemas socioeconómicos & $3.20(.55)$ & $3.11(.60)$ & $3.07(.52)$ & 1.49 & .23 & - \\
\hline
\end{tabular}

Nota: *indica diferencias estadísticamente significativas $\mathrm{p}<.05$.

Tabla 6. Influencia de la práctica del deporte en la percepción sobre los impactos de la Maratón.

\begin{tabular}{|c|c|c|c|c|c|}
\hline & $\begin{array}{c}\text { Sí } \\
\text { M (DT) }\end{array}$ & $\begin{array}{c}\text { No } \\
M \text { (DT) }\end{array}$ & $\mathbf{T}$ & P valor & d Cohen \\
\hline Factor 1 - Beneficios económicos e imagen de la ciudad & $3.84(.46)$ & $3.77(.59)$ & 1.38 & .17 & - \\
\hline Factor 2 - Beneficios sociales & $3.15(.46)$ & $2.99(.57)$ & 2.33 & $.002 * *$ & .31 \\
\hline Factor 3 - Orgullo e identificación con la comunidad & $3.45(.66)$ & $3.39(.69)$ & .88 & .38 & - \\
\hline Factor 4 - Impacto deportivo & $3.41(.64)$ & $3.25(.73)$ & 1.66 & $.02 *$ & .24 \\
\hline Factor 5 - Problemas socioeconómicos & $3.15(.49)$ & $3.06(.63)$ & 3.00 & .10 & - \\
\hline
\end{tabular}

Nota: **indica diferencias estadísticamente significativas $p<.002 ;{ }^{*} p<.05$.

Tabla 7. Correlaciones entre la edad y los impactos percibidos sobre la Maratón.

\begin{tabular}{lc}
\hline & Edad \\
\hline Factor 1 - Beneficios económicos e imagen de la ciudad & -.04 \\
Factor 2 - Beneficios sociales & .03 \\
Factor 3 - Orgullo e identificación con la comunidad & -.03 \\
Factor 4 - Impacto deportivo & .01 \\
Factor 5 - Problemas socioeconómicos & $.15^{\star}$ \\
\hline
\end{tabular}

Nota: *indica diferencias estadísticamente significativas $p<.01$.

tividad física tienden a valorar más positivamente los diferentes factores, incluido el de problemas socioeconómicos. No obstante, solo se observaron diferencias estadísticamente significativas $(\mathrm{p}<.05)$ en el factor de beneficios sociales y en el de impacto deportivo.

Por último, se comprobó la relación entre la edad y la percepción sobre los impactos de la Maratón mediante un análisis de correlación. El análisis de correlación solo detectó una correlación estadísticamente significativa $(p<.05)$ entre la edad y el factor de problemas socioeconómicos $(r=.15)$, observándose que a mayor edad existe una tendencia a valorar de forma más acusada los posibles problemas socioeconómicos derivados de la acogida del evento.

\section{Discusión}

En este trabajo se presenta una serie de indicadores para medir la percepción social sobre un evento deportivo de carácter regular. Los análisis realizados sobre los indicadores propuestos muestran la agrupación de los indicadores en cinco factores relacionados con el constructo del impacto social de los eventos deportivos de carácter regular y participativo.

El primer factor está relacionado con los beneficios económicos y la imagen de la ciudad, mostrando una puntuación positiva en la mayoría de los indicadores. En este sentido, los residentes entrevistados parecen percibir positivamente la repercusión del Maratón de Valencia en aspectos de carácter económico y de mejora 
de la imagen de la ciudad. En estudios previos ya se ha apuntado que los eventos deportivos regulares de carácter participativo pueden contribuir a generar beneficios en las localidades que los acogen debido a una menor inversión en infraestructuras e instalaciones y a la asistencia de participantes de fuera de la localidad, que generan un gasto turístico en la localidad (Ouyang et al., 2019; Taks, 2013). El Maratón de Valencia atrae numerosos participantes tanto nacionales como extranjeros que supone un gasto turístico directo en la ciudad de 23 millones de euros, implicando que cada euro invertido por la organización se tradujo en 4.2 euros en gasto turístico realizado por los corredores y sus acompañantes durante su estancia en la última edición del evento (Maudos et al., 2020). Por otro lado, el éxito en la organización se ve reflejado en el elevado grado de satisfacción de los participantes (8.9 de media sobre 10), siendo evaluado con sobresaliente por el 75\% de los corredores (Maudos et al., 2020), hecho que permite mejorar la imagen del evento y de la ciudad como organizadora de eventos deportivos.

Por otro lado, se identificó un factor relacionado con los beneficios sociales en el que se integran indicadores como las oportunidades para trabajar, la distribución equilibrada de los beneficios en el municipio, la oportunidad para conocer a otras personas, la inclusión de personas con discapacidad o de grupos en riesgo de exclusión social. De acuerdo, con las consideraciones de trabajos previos este tipo de eventos pueden suponer mayores beneficios sociales que los mega eventos deportivos (Taks, 2013).

El tercer factor está relacionado con el orgullo de los residentes por ser los anfitriones del evento, el incremento de la satisfacción consigo mismos y con el conjunto de la sociedad y el sentimiento de pertenencia al municipio. Cuando se compara un mega evento con un evento de pequeña o mediana escala, parece que puede permitir más intercambio en la comunidad local y, por lo tanto, es más probable que genere resultados que beneficien más a esa comunidad (Taks, Chalip, et al., 2015). De acuerdo con Ouyang et al. (2019), los eventos deportivos regulares pueden contribuir a generar impactos socioculturales positivos relacionados con el sentimiento de pertenencia, identificación y orgullo de la comunidad. Los eventos deportivos pueden crear un sentimiento de comunidad y unión que permite a los individuos más aislados sentirse conectados a algo (Taks, Littlejohn, et al., 2015). Este sentimiento se suele denominar "cohesión social" y puede aumentar significativamente la calidad de vida y el bienestar (e.g., Heere et al., 2013; Kaplanidou et al., 2013; Klein, 2013).

El cuarto factor hace referencia al impacto deportivo en términos de participación, apoyo y fomento del deporte en diversos colectivos de la ciudad. Este factor es valorado por los residentes entrevistados con una tendencia positiva en la mayoría de los indicadores. Una de las ventajas de los eventos deportivos regulares y participativos es la posibilidad que ofrecen a la población local de participar en los mismos como deportistas, voluntarios u organizadores (Taks, 2013). Esto puede repercutir en la mejora de la calidad de vida de los residentes, así como en el grado de identificación con la localidad y el evento (Parra-Camacho et al., 2020). No obstante, es importante destacar que no existe una evidencia clara de que la celebración de un evento deportivo produzca un incremento en la práctica deportiva entre los residentes locales, pero se ha destacado su capacidad para aumentar la práctica deportiva alentando a que la población sea más activa físicamente (Hautbois et al., 2020). Aunque el caso de los eventos participativos es obviamente diferente en cuanto que inducen una participación directa (Hautbois et al., 2020).

Por último, los indicadores relacionados con posibles impactos negativos quedan recogidos en el factor de problemas socioeconómicos. Los residentes destacan como principal problema las posibles alteraciones en la vida diaria por los problemas del tráfico, la congestión o el ruido y la aglomeración o acumulación desordenada de personas durante la prueba. Estos eventos no están exentos de impactos negativos o costes, que en el caso del Maratón de Valencia están relacionados principalmente con el corte de calles, la restricción de la circulación por las zonas del circuito y el posible ruido en las zonas de animación de la carrera. No obstante, los posibles costes siempre son muy inferiores a los generados por los mega eventos deportivos (Taks, 2013).

Por lo tanto, se puede apreciar un intercambio social positivo entre los residentes entrevistados ya que los beneficios del evento, expresados en los cuatro primeros factores de la escala, son valorados con una tendencia positiva o neutral, mientras que los costes no son tan destacados como algunos de los indicadores relacionados con los beneficios. De acuerdo, con la TIS si los beneficios superan a los costes se puede considerar que el intercambio es positivo $y$, por lo tanto, puede existir una mayor probabilidad de respaldar la celebración del evento (Gursoy et al., 2017; Waitt, 2003).

Respecto a las diferencias según diversas variables sociodemográficas, solo se observaron en determinados factores como los beneficios económicos y la mejora de la imagen en el caso del género, con una puntuación ligeramente más alta en los hombres. También, según el nivel de estudios, en el factor de impacto en el deporte con puntuaciones algo más altas para los residentes con nivel de estudios universitarios. En estudios previos se han encontrado diferencias esta- 
dísticamente significativas en las percepciones de los residentes sobre los impactos en función del género (Añó et al., 2012; S. S. Kim \& Petrick, 2005; Ritchie et al., 2009) y también en función del nivel educativo (Ma et al., 2013), aunque estos estudios analizan eventos de características diferentes y en algunos casos se trata de mega eventos o eventos que no se celebran anualmente en la misma localización. Por esta razón es necesario seguir analizando la influencia de estas variables en la percepción sobre los impactos en eventos de menor escala y de carácter regular.

En cuanto a las diferencias según la práctica deportiva, se comprobó que los residentes que practicaban el deporte relacionado con el evento tendían a valorar más positivamente los impactos del evento. Esto puede tener una interpretación lógica por la vinculación e identificación con el evento, aspecto que puede determinar una mejor valoración de los impactos del evento en la ciudad y un mayor conocimiento e interés por la prueba (Chen et al., 2018). Para finalizar con las variables sociodemográficas, se observa una correlación pequeña entre la edad y el factor de problemas socioeconómicos, que indica que a mayor edad se incrementa la valoración de los costes del evento. En trabajos previos, la edad de los residentes ha sido una variable que ha afectado a la percepción de los impactos (Añó et al., 2012; S. S. Kim \& Petrick, 2005; Lee et al., 2013; Ritchie et al., 2009), aunque los resultados han sido diferentes según el estudio y no existe un consenso ya que pueden estar condicionados por las características sociales y culturales de la población analizada.

Es importante destacar que este trabajo tiene una serie de limitaciones que se deben tener en cuenta a la hora de interpretar los resultados. En primer lugar, se ha utilizado un muestreo de conveniencia que no es representativo de la población residente en la ciudad de Valencia, por lo que se debe ser cauto a la hora de generalizar los resultados al conjunto de los residentes. En este sentido, en futuros trabajos se deberá intentar recoger una muestra representativa de la población objeto de estudio de acuerdo con las principales variables sociodemográficas. Por otro lado, aunque el análisis paralelo del AFE recomendó la agrupación en cinco factores, se ha comprobado que la fiabilidad de algunas de las dimensiones no presenta valores óptimos de acuerdo con las recomendaciones de la literatura. Asimismo, fue necesario eliminar numerosos indicadores de la propuesta inicial (diez) ya que no cumplían con los criterios recomendados por la literatura. Por esta razón, se debe comprobar en otras muestras las propiedades psicométricas de los indicadores y su agrupación en los factores identificados, analizando la fiabilidad y valorando la reespecificación del modelo. También será necesario comprobar con un análisis factorial confirmatorio la solución factorial propuesta por el AFE y explorar la posibilidad de identificar factores de primer y segundo orden.

\section{Conclusiones}

Este trabajo realiza una contribución en la literatura sobre el impacto social de los eventos deportivos participativos y regulares de pequeña y mediana escala, identificando posibles dimensiones e indicadores que permitan a los organizadores medir el impacto social en los municipios que los acogen. No obstante, es necesario realizar más trabajos en esta área, con muestras representativas y eventos de características similares, para confirmar la agrupación en factores con características similares a los identificados en este trabajo. El intercambio social positivo que genera la celebración del Maratón en la ciudad de Valencia, de acuerdo con el análisis de la percepción de los residentes entrevistados, permite reforzar la buena gestión del impacto social del evento por parte de los organizadores y de la administración pública. En cualquier caso, y como se ha indicado previamente, es necesario realizar más estudios de impacto social de este evento para poder generalizar los resultados al conjunto de la población y comprobar la evolución de la percepción de los ciudadanos a lo largo del tiempo.

\section{B I B LI O G R A FÍA}

Añó, V., Calabuig, F., \& Parra, D. (2012). Social impact of a major sport event: The Formula 1 Grand Prix of Europe. Cultura, Ciencia y Deporte, 7(19), 53-65. https://doi.org/10.12800/ccd.v7i19.23

Chen, K.-C., Gursoy, D., \& Lau, K. L. K. (2018). Longitudinal impacts of a recurring sport event on local residents with different level of event involvement. Tourism Management Perspectives, 28, 228-238. https:// doi.org/10.1016/j.tmp.2018.09.005

Chien, P. M., Ritchie, B. W., Shipway, R., \& Henderson, H. (2012). I Am Having a Dilemma Factors Affecting Resident Support of Event Development in the Community. Journal of Travel Research, 51(4), 451463. https://doi.org/10.1177/0047287511426336
Chou, C.-P., \& Bentler, P. M. (1995). Estimates and tests in structural equation modeling. En Structural equation modeling: Concepts, issues, and applications (pp. 37-55). Sage Publications, Inc.

Daniels, M. J., \& Norman, W. C. (2003). Estimating the Economic Impacts of Seven Regular Sport Tourism Events. Journal of Sport \& Tourism, 8(4), 214-222. https://doi.org/10.1080/1477508032000161528

Djaballah, M., Hautbois, C., \& Desbordes, M. (2015). Non-mega sporting events' social impacts: A sensemaking approach of local governments' perceptions and strategies. European Sport Management Quarterly, 15(1), 48-76. https://doi.org/10.1080/16184742.2014.1 000353 
Duan, Y., Mastromartino, B., Zhang, J. J., \& Liu, B. (2020). How do perceptions of non-mega sport events impact quality of life and support for the event among local residents? Sport in Society, 0(0), 1-20. https://doi.org/10.1080/17430437.2020.1804113

Getz, D., Svensson, B., Pettersson, R., \& Gunnervall, A. (2012). Hallmark events: Definition, goals and planning process. International Journal of Event Management Research, 7(1/2), 47-67.

Gibson, H. J., Kaplanidou, K., \& Kang, S. J. (2012). Small-scale event sport tourism: A case study in sustainable tourism. Sport Management Review, 15(2), 160-170. https://doi.org/10.1016/j.smr.2011.08.013

Gibson, H. J., Willming, C., \& Holdnak, A. (2003). Small-scale event sport tourism: Fans as tourists. Tourism Management, 24(2), 181-190. https://doi.org/10.1016/S0261-5177(02)00058-4

Gursoy, D., Milito, M. C., \& Nunkoo, R. (2017). Residents' support for a mega-event: The case of the 2014 FIFA World Cup, Natal, Brazil. Journal of Destination Marketing \& Management, 6(4), 344-352. https:// doi.org/10.1016/j.jdmm.2017.09.003

Gursoy, D., Ouyang, Z., Nunkoo, R., \& Wei, W. (2019). Residents' impact perceptions of and attitudes towards tourism development: A metaanalysis. Journal of Hospitality Marketing \& Management, 28(3), 306333. https://doi.org/10.1080/19368623.2018.1516589

Hair, J. F., Black, W. C., Babin, B. J., \& Anderson, R. E. (2014). Multivariate Data Analysis (7th edition). Pearson.

Hautbois, C., Djaballah, M., \& Desbordes, M. (2020). The social impact of participative sporting events: A cluster analysis of marathon participants based on perceived benefits. Sport in Society, 23(2), 335-353. https://doi.org/10.1080/17430437.2019.1673371

Heere, B., Walker, M., Gibson, H., Thapa, B., Geldenhuys, S., \& Coetzee, W. (2013). The power of sport to unite a nation: The social value of the 2010 FIFA World Cup in South Africa. European Sport Management Quarterly, 13(4), 450-471. https://doi.org/10.1080/16184742. 2013.809136

Higham, J. (1999). Commentary - Sport as an Avenue of Tourism Development: An Analysis of the Positive and Negative Impacts of Sport Tourism. Current Issues in Tourism, 2(1), 82-90. https://doi. org/10.1080/13683509908667845

Kaplanidou, K. (Kiki), Karadakis, K., Gibson, H., Thapa, B., Walker, M., Geldenhuys, S., \& Coetzee, W. (2013). Quality of Life, Event Impacts, and Mega-Event Support among South African Residents before and after the 2010 FIFA World Cup. Journal of Travel Research, 52(5), 631645. https://doi.org/10.1177/0047287513478501

Kim, H. J., Gursoy, D., \& Lee, S.-B. (2006). The impact of the 2002 World Cup on South Korea: Comparisons of pre- and post-games. Tourism Management, 27(1), 86-96. https://doi.org/10.1016/j.tourman.2004.07.010

Kim, S. S., \& Petrick, J. F. (2005). Residents' perceptions on impacts of the FIFA 2002 World Cup: The case of Seoul as a host city. Tou rism Management, 26(1), 25-38. https://doi.org/10.1016/j.tourman.2003.09.013

Klein, C. (2013). Social Capital or Social Cohesion: What Matters For Subjective Well-Being? Social Indicators Research, 110(3), 891-911. https://doi.org/10.1007/s11205-011-9963-x

Lee, S.-B., Lee, C.-K., Kang, J., Lee, E.-Y., \& Jeon, Y. J. J. (2013). Residents' Perception of the 2008 Beijing Olympics: Comparison of Pre and Post-Impacts. International Journal of Tourism Research, 15(3), 209-225. https://doi.org/10.1002/jtr.885

Lloret-Segura, S., Ferreres-Traver, A., Hernández-Baeza, A., \& TomásMarco, I. (2014). El análisis factorial exploratorio de los ítems: Una guía práctica, revisada y actualizada. Anales de Psicología / Annals of Psychology, 30(3), 1151-1169. https://doi.org/10.6018/analesps.30.3.199361

Ma, S. C., Ma, S. M., Wu, J. H., \& Rotherham, I. D. (2013). Host residents' perception changes on major sport events. European Sport Management Quarterly, 13(5), 511-536. https://doi.org/10.1080/16184742. 2013.838980

Martínez, J. M., López, J. A., Norte, A., \& Martinez-Rodriguez, A (2019). Dietary and nutritional approach to do the Rioja Bike Race: Case study. (Enfoque dietético-nutricional para afrontar la Rioja Bike Race: caso práctico). Cultura, Ciencia y Deporte, 14(41), 107-111. https://doi.org/10.12800/ccd.v14i41.1270
Maudos, J., Aldás, J., Benages, E., \& Zaera, I. (2020). 39 Maratón Trinidad Alfonso de València. Impacto económico y valoración de los corredores. Instituto Valenciano de Investigaciones Económicas. https://www.ivie. es/es_ES/ptproyecto/impacto-economico-del-39-maraton-trinidadalfonso-valencia/

Ntloko, N. J., \& Swart, K. (2008). Sport tourism event impacts on the host community - a case study of Red Bull Big Wave Africa. South African Journal for Research in Sport, Physical Education and Recreation, 30(2), 79-93. https://doi.org/10.4314/sajrs.v30i2.25991

O'Brien, D., \& Chalip, L. (2007). Executive training exercise in sport event leverage. International Journal of Culture, Tourism and Hospitality Research, 1(4), 296-304. https://doi.org/10.1108/17506180710824181

Oshimi, D., \& Harada, M. (2019). Host residents' role in sporting events: The city image perspective. Sport Management Review, 22(2), 263275. https://doi.org/10.1016/j.smr.2018.04.002

Ouyang, Z., Gursoy, D., \& Chen, K.-C. (2019). It's all about life: Exploring the role of residents' quality of life perceptions on attitudes toward a recurring hallmark event over time. Tourism Management, 75, 99-111. https://doi.org/10.1016/j.tourman.2019.04.032

Parra-Camacho, D., González-García, R. J., \& Alonso-Dos-Santos, M. (2020). Social impact of a participative small-scale sporting event. Sport, Business and Management: An International Journal, ahead-ofprint(ahead-of-print). https://doi.org/10.1108/SBM-12-2019-0119

Parra-Camacho, D., González-García, R. J., Añó, V., \& Ayora, D. (2016). Visitors' perception on the social impact and intentions regarding holding a small-scale sporting event. Revista de Psicología del Deporte, 25(3, Supl 1), 93-96

Prayag, G., Hosany, S., Nunkoo, R., \& Alders, T. (2013). London residents' support for the 2012 Olympic Games: The mediating effect of overall attitude. Tourism Management, 36, 629-640. https://doi. org/10.1016/j.tourman.2012.08.003

Ritchie, B. W., Shipway, R., \& Cleeve, B. (2009). Resident Perceptions of Mega-Sporting Events: A Non-Host City Perspective of the 2012 London Olympic Games. Journal of Sport \& Tourism, 14(2-3), 143-167. https://doi.org/10.1080/14775080902965108

Sánchez-Sáez, J. A., Segado-Segado, F., \& Vidal, A. (2018). Sports events socially responsible as the engine for local development. Journal of Sports Economics \& Management, 8(3), 172-186.

Taks, M. (2013). Social sustainability of non-mega sport events in a global world. EJSS. European Journal for Sport and Society, 10(2), 121-141.

Taks, M., Chalip, L., \& Green, B. C. (2015). Impacts and strategic outcomes from non-mega sport events for local communities. European Sport Management Quarterly, 15(1), 1-6. https://doi.org/10.1080/16 184742.2014.995116

Taks, M., Littlejohn, M., Wiood, L., \& Snelgrove, R. (2015). Construct validity of social impact scales for sport events. Human Kinetics $\mathrm{Pu}$ blications, 1-13

Tanaka, J. S., \& Huba, G. J. (1989). A general coefficient of determination for covariance structure models under arbitrary GLS estimation. British Journal of Mathematical and Statistical Psychology, 42(2), 233239. https://doi.org/10.1111/j.2044-8317.1989.tb00912.x

Timmerman, M. E., \& Lorenzo-Seva, U. (2011). Dimensionality assessment of ordered polytomous items with parallel analysis. Psychological Methods, 16(2), 209-220. https://doi.org/10.1037/ a0023353

Vegara-Ferri, J. M., Angosto, S., \& Parra-Camacho, D. (2020). Efecto de la satisfacción de los residentes entre los impactos percibidos y las intenciones futuras respecto a la celebración de un evento de pequeña escala. Revista iberoamericana de psicología del ejercicio y el deporte, 15(1), 81-91.

Veltri, F. R., Miller, J., \& Harris, A. (2009). Club sport national tournament: Economic impact of a small event on a mid-size community. Recreational Sports Journal, 33(2), 119-128. CABDirect2.

Waitt, G. (2003). Social impacts of the Sydney Olympics. Annals of Tourism Research, 30(1), 194-215. https://doi.org/10.1016/S01607383(02)00050-6

Wilson, R. (2006). The economic impact of local sport events: Significant, limited or otherwise? A case study of four swimming events. Managing Leisure, 11(1), 57-70. https://doi.org/10.1080/13606710500445718 